\title{
O CUIDADO ESPECIALIZADO DO EGRESSO DA RESIDÊNCIA EM ENFERMAGEM DO INSTITUTO NACIONAL DE CÂNCER - INCA
}

\author{
The Specialized Care of the Egress from the Residence in Nursing \\ of the National Institute of Cancer - INCA \\ La Atención Especializada del Egreso de la Residencia en \\ Enfermería del Instituto Nacional del Cáncer - INCA
}

Carlos Joelcio de Moraes Santana

Gertrudes Teixeira Lopes

\begin{abstract}
Resumo
0 objeto do estudo é o cuidado de enfermagem prestado pelos egressos da residência em enfermagem do INCA. Com objetivo de caracterizar a prática dos egressos da residência em enfermagem no CEMO (Centro de Transplante de Medula Óssea). Utilizaram-se os conceitos de especialista de Patrícia Benner e da United Kingdom Council Center (UKCC). Trata-se de uma pesquisa qualitativa realizada no CEMO em julho de 2004. Os sujeitos do estudo foram nove enfermeiros egressos da residência em enfermagem, da área de oncologia clínica, atuantes no CEMO desde 1991, data do início das contratações dos egressos. Utilizou-se a técnica de Grupo Focal para coletar os dados. A análise seguiu a orientações de análise temática, segundo Minayo. Os resultados evidenciaram a categoria "Cuidado Especializado do Egresso da Residência", que abrangeu temas relacionados com protocolo/tratamento/farmacologia, prescrição de enfermagem, orientação e experiência, e esperança e complexidade. Concluiu-se, portanto, que o trabalho realizado pelos egressos da residência de enfermagem no CEMO é especializado.
\end{abstract}

Palavras-chave: Enfermagem. Internato e Residência. Especialização. Enfermagem Oncológica. Transplante de Medula Óssea.

\begin{abstract}
The object of this study is the nursing care rendered by egress from nursing residence of INCA. With the objective of characterize the experience of the egress from nursing residence in the CEMO. It was utilized the specialist concepts of Patrícia Benner and from United Kingdom Council Center (UKCC). Qualitative Research realized in the CEMO in july of 2004. The subjects of the study were nine nurses egress from nursing residence, of the clinical oncology area, working in the CEMO since 1991, beginning date of the egress contract. The Focal Group technique was used to the data collection. The analysis followed the theme analysis guidance according to Minayo. The results evidenced the category "Specialized Care of the Egress from the Residence", which included subjects related to protocol/ treatment/pharmacology, nursing prescription, orientation and experience, and hope and complexity. It was concluded that the work realized by the egress from nursing residence in the CEMO is specialized.
\end{abstract}

Keywords: Nursing. Intership and Residency. Specialism. Oncologic Nursing. Bone Marrow Transplantation.

\section{Resumen}

El objeto del estudio es el cuidado de enfermería prestado por los egresos de la residencia en enfermería del INCA. El objetivo fue caracterizar la práctica de los egresos de la residencia en enfermería en el CEMO (Centro de Trasplante de Medula Ósea). Se utilizaron los conceptos de especialista de Patrícia Benner y de la United Kingdom Council Center (UKCC). Pesquisa cualitativa realizada en el CEMO en julio de 2004. Los sujetos del estudio fueron nueve enfermeros egresos de la residencia en enfermería, del área de oncología clínica, actuantes en el CEMO desde 1991, fecha del inicio de contrataciones de los egresos. Fue utilizada la técnica del Grupo Focal para colectar los datos. Se utilizó la técnica de análisis temática, en la perspectiva de Minayo. Fue identificada la categoría "El Cuidado Especializado del Egreso de la Residencia", que abarcó temas relacionados con protocolo/ tratamiento/farmacología, prescripción de enfermería, orientación y experiencia, y esperanza y complejidad. Se concluyó que el trabajo realizado por los egresos de la residencia de enfermería en el CEMO es especializado.

Palabras clave: Enfermería. Internato y Residencia. Especialización. Enfermería Oncológica. Trasplante de Médula Ósea. 


\section{CONSIDERAÇÕES INICIAIS}

A História da Enfermagem Oncológica tem seu início nos Estados Unidos. Como especialidade, nas últimas décadas, mostra um grande progresso da prática profissional, especialmente no cuidado do paciente com uma doença sistêmica e um extraordinário e crescente entendimento do câncer como um problema biológico'.

As enfermeiras especialistas desempenhavam papéis importantes no cuidado de "cabeceira", proporcionando medidas de conforto para pacientes cirúrgicos e tratamento paliativo para os pacientes terminais ${ }^{1}$. A atuação do enfermeiro em oncologia cresceu com o advento dos ensaios clínicos conduzidos com novos agentes terapêuticos quimioterápicos. Estes ensaios clínicos trouxeram a necessidade de um trabalho conjunto da equipe multidisciplinar voltada para o cuidado do paciente com câncer e a pesquisa'.

Assim, no início dos anos de 1970, os ensaios clínicos e a equipe multidisciplinar se estenderam da academia para a comunidade, crescendo, então, a demanda para a enfermagem oncológica, o que estimulou o desenvolvimento desta especialidade, culminando, assim, com o aparecimento das organizações de enfermagem oncológica, da educação curricular e dos cursos de especialização, de atualização e de outros. Um reduzido, mas logo crescente número de enfermeiras, trabalhando inicialmente em centros de pesquisa, deu início às discussões, levando à criação da Oncology Nursing Society (ONS), em 1975, que é a maior organização científica do mundo na especialidade do câncer, promovendo fóruns de discussões e constituindo um meio pelo qual as enfermeiras podem contribuir para a especialidade ${ }^{1}$.

No Brasil, a organização das enfermeiras na área oncológica inicia-se em 1983, por ocasião do XXXI Congresso Brasileiro de Enfermagem, realizado em São Paulo. Nesta oportunidade, em uma reunião de enfermeiras da área oncológica, desencadeou-se o processo de organização da categoria em nível nacional, que culminou, em 1984, com a criação da Sociedade Brasileira de Enfermagem Oncológica do Estado de São Paulo (SEOESP). Em 1988, em Salvador, aconteceu a eleição e posse da primeira diretoria da Sociedade Brasileira de Enfermagem Oncológica (SBEO)².

Com o desenvolvimento da Enfermagem Oncológica no país e em especial no INCA, órgão que se configura como referência no controle do câncer, na formação de profissionais especializados e de fomento de pesquisa na área, cresce a idéia da criação de um programa que pudesse especializar 0 enfermeiro no Instituto, devido à escassez de especialistas na área. Nesta perspectiva, os enfermeiros do INCA, em 1985, elaboraram um projeto para suprir essa carência, visto que 0 ensino da cancerologia na graduação era praticamente inexistente. Tal iniciativa contou com apoio da Chefe de Enfermagem da Instituição Sílvia Beatriz de Assis, assim como do Diretor Geral do INCA, Dr. Geraldo Matos de Sá . 0 Projeto foi aprovado em 27 de dezembro de 1985 pelo Diretor Nacional de Doenças Crônico-Degenerativas, Dr. Geniberto Paiva Campos².
0 Projeto de Residência em Enfermagem do INCA, então aprovado pelo Ministério da Saúde no processo MS 0032/86, tendo como Diretor Geral o Dr. Walter Roriz de Carvalho iniciou suas atividades em 02 de junho de 1986, com cinco residentes, adotando a Instrução Normativa do DASP n ${ }^{0}$ 52-31/3176, sendo definida como curso de nível de especialização destinado a enfermeiros com certificado de graduação em enfermagem, caracterizado por treinamento em serviço, funcionando sob responsabilidade da instituição com aprovação oportuna pelos órgãos competentes ${ }^{3}$.

Os objetivos eram o de proporcionar ao enfermeiro conhecimento técnico-científico na área oncológica de forma global e específica; formar especialistas em assistência de enfermagem ao paciente oncológico em todos os níveis (prevenção, tratamento e reabilitação); formar profissionais de modo a torná-los elementos multiplicadores em oncologia em nível hospitalar e saúde comunitária3.

0 Programa era implementado em 2 anos com 40 horas semanais de treinamento, totalizando 3.840 horas, distribuídas em: $20 \%$ de teoria ( 768 horas) e $80 \%$ de atividades práticas (3.072 horas) e oferecia como áreas de concentração: a oncologia clínica, a oncologia cirúrgica e a especializada, que era composta pelo Centro de Tratamento Intensivo, Centro Cirúrgico, Central de Material e Unidade Intermediária4.

Em 1990, a Campanha Nacional de Combate ao Câncer, órgão do Ministério da Saúde que administrava financeiramente e apoiava o INCA, foi extinta, e a insuficiência de servidores ameaçava o funcionamento de várias unidades. Sem recursos humanos suficientes e sem concursos públicos para repô-los, não havia como completar as vagas abertas por aposentadorias, transferências, falecimentos e demissões espontâneas dos servidores.

A situação da Instituição se agravava. Procurando dar solução para o problema, um grupo de quatro médicos, entre eles 0 então Diretor do Instituto Nacional de Câncer, Marcos Fernando de Oliveira Moraes, inspiraram-se na mesma estratégia adotada por outras instituiç̃es de renome e criaram a Fundação Ary Frauzino (FAF). 0 nome Ary Frauzino foi escolhido em homenagem ao cancerologista que foi diretor do INCA entre os anos de 1980 e 1985. Foi então inaugurada em 19 de dezembro de 1991. 0 registro da Fundação na Procuradoria Geral da Justiça do Rio de Janeiro foi realizado em 9 de abril de 1991. A principal receita da FAF advém do Sistema Único de Saúde, que remunera os serviços de assistência oncológica prestados pelo INCA ${ }^{5}$.

Assim, o processo seletivo foi possibilitado através da Fundação Ary Frauzino (FAF) para a Pesquisa e Controle do Câncer. Em dezembro de 1991, foram realizadas as primeiras contratações de enfermeiros para o Instituto, com uma política voltada para o aproveitamento dos egressos da Residência em Enfermagem Oncológica. Atualmente os enfermeiros egressos da Residência correspondem a 32,34\% dos enfermeiros do Instituto.

0 Centro de Transplante de Medula Ósseo (CEMO), inaugurado em 1983, foi o pioneiro na contratação dos egressos da Residência em Enfermagem Oncológica. A unidade se destaca hoje como um centro de excelência e de referência para Transplante de Medula Óssea alogeneico e autólogo no Brasilit. 
Dada a importância, a especificidade e a complexidade do cuidado implementado, necessitando, portanto, de profissionais com formação especializada na área, e levandose em consideração que os egressos da residência representam $42,5 \%$ do total de enfermeiros desta unidade em seu quadro de pessoal, parte-se do pressuposto que a assistência de enfermagem prestada pelos egressos da Residência em Enfermagem do INCA é especializada.

Nesta perspectiva, definiu-se como objeto do estudo: 0 cuidado especializado doa egressos da Residência em Enfermagem no CEMO, e como objetivo geral caracterizar a prática dos egressos da Residência em Enfermagem, realizada no Centro de Transplante de Medula Óssea (CEMO). Os objetivos específicos foram analisar a prática de enfermagem realizada pelos egressos da residência no CEMO e discutir a assistência de enfermagem prestada pelos egressos da Residência, no CEMO.

Desta forma, o estudo contribui na medida em que ratificamos a importância da formação especializada para um cuidado de excelência, abrangendo não só aspectos tecnicistas, mas também valores humanísticos na implementação da assistência.

\section{REFERÊNCIAL TEÓRICO}

Os referenciais teóricos adotados neste estudo foram os conceitos de especialista delineados por Patrícia Benner e pela UKCC (United Kingdom Council Center).

Para as enfermeiras adquirirem competência, passam necessariamente por cinco estágios: Iniciada, Iniciada Avançada, Competente, Proficiente e Especialista ${ }^{6}$.

As Enfermeiras Especialistas dedicam-se a um campo específico de atuação, têm grande experiência, compreendem de maneira intuitiva cada situação, não se perdendo em soluções e diagnósticos infundados ${ }^{6}$.

As Enfermeiras Especialistas não são difíceis de serem identificadas, pois dão opiniões clínicas ou gerenciam situações complexas de forma exemplar. São também consultadas por outras enfermeiras e podem ser eficazes quando se pede uma avaliação médica mais amiúde ao se detectarem precocemente algumas alterações clínicas. Ainda estabelecem estratégias a fim de melhorarem a qualidade do cuidado prestado aos pacientes em determinadas áreas do saber. Atuam sempre de forma ética para que se mantenha o respeito pela categoria. As especialistas elaboram estratégias objetivando estabelecer um papel referencial quanto à gestão, educação e pesquisa relevantes. Estão sempre em busca de atualização, objetivando sempre o desenvolvimento da categoria. Existe autonomia para tomada de decisões dentro de suas competências estabelecidas. Dentre outras características estão também as de comunicação, utilizando a metodologia para utilização do processo de enfermagem ${ }^{6}$.

0 Conselho Central do Reino Unido (UKCC) define abaixo as competências dos enfermeiros em assuntos como 0 cuidado, complementando os conceitos anteriormente referidos, discorrendo sobre alguns pontos quanto à atuação das enfermeiras especialistas? ${ }^{7}$.
Competências nucleares - cuidados básicos nos princípios da melhor prática; cuidado baseado na experiência de conhecimento especializado; 0 conhecimento especializado deve ser utilizado em conjunto com avançadas técnicas de avaliação, de apresentar subsídios que contribuam com o cuidado multidisciplinar; capacidade de desfiar e melhorar a prática atual; ser capaz de atuar como agente de mudança, planejando, investigando e avaliando a mudança; capacidade de potencializar, através do conhecimento, capacidades e competência demonstrável.

Gestão - a capacidade de gerir a interface do cuidado dentro do contexto da especialidade; capacidade de gerir um serviço, fornecendo cuidados de alta qualidade baseados na investigação, considerando recursos existentes; participar das políticas multidisciplinares tendo em vista a especialização e as linhas de orientação para uso no serviço; tomar responsabilidade para o seu próprio desenvolvimento pessoal e profissional; estar envolvido no desenvolvimento do serviço.

Consultoria - atuar como uma fonte especialista na gestão dos clientes em todo o serviço no âmbito dos assuntos específicos; fornecer aconselhamento especializado, referente à compra e provisionamento de recursos e serviços pertencentes à especialidade; contribuir para a supervisão clínica e revisão ao nível do grupo no serviço. Formação - identificar as necessidades formativas do pessoal, clientes e pessoas que deles cuidam, fazendo recomendações quando houver necessidade; dar contribuição especializada; identificar as suas próprias necessidades educacionais, de forma a manter a especialização; ser responsável pelo seu próprio desenvolvimento profissional.

Investigação/Auditoria - ser pró-ativo em estudos correntes, de forma a avaliar a eficácia do serviço; participar ativamente de projetos de investigação em seu âmbito clínico; avaliar de forma crítica os resultados da investigação.

\section{METODOLOGIA}

0 estudo do tipo qualitativo foi realizado no Centro de Transplante de Medula Óssea do Instituto Nacional de Câncer (CEMO/ INCA), localizado no $7^{\circ}$ andar do prédio situado na Praça Cruz Vermelha 23, Centro, na Cidade do Rio de Janeiro.

Os sujeitos do estudo foram os enfermeiros egressos do Programa de Residência em Enfermagem, da área de Oncologia Clínica, atuantes no CEMO desde 1991, marco inicial das contratações dos egressos, até 2002, ano em que foram feitas as últimas contratações. Portanto, incluindo-se o período de Residência, egressos com quatro a quinze anos de atuação na área.

Os sujeitos foram convidados a participar da pesquisa mediante carta-convite, acompanhada do Termo de Consentimento Livre e Esclarecido, elaborado após a aprovação do Comitê de Ética e Pesquisa do INCA, informando o objetivo do encontro, a importância da presença, a data, a hora, o local e a duração do evento. Foram convidados 19 enfermeiros que atuam tanto na Unidade de Internação, na Unidade de Pacientes 
Externos e no Banco de Sangue de Cordão Umbilical e Placentário (BSCUP). Participaram da pesquisa enfermeiros do serviço diurno e noturno. Do conjunto de convidados, nove compareceram, sendo seis da unidade de internação, dois do ambulatório e um da chefia.

Dentre os que não atenderam, dois estavam de licença, um estava de férias e sete estavam impossibilitados de comparecer, devido a outros compromissos.

A moderadora informou aos sujeitos que o debate seria gravado em fita magnética e solicitou que escolhessem um nome fictício para preservarem suas identidades. Os participantes optaram, portanto, por nomes de flores.

Para a coleta das informações, utilizou-se a técnica de grupo focal. Esta técnica utilizada na pesquisa qualitativa tem como objetivo coletar dados da vida cotidiana, em uma determinada população, abordando assuntos de interesse comum.

A Técnica de Grupo Focal busca a informação não individual, mas sim de um grupo. Os grupos focais facilitam os entrevistados a expressar seus próprios centros de atenção e de suas próprias reações aos conceitos que são importantes para eles ${ }^{8}$.

Grupo Focal é um debate aberto e acessivel a todos; os assuntos em questão são de interesse em comum; a diferença de status entre os participantes não é levada em consideração, e o debate se fundamenta em uma discussão racional. 0 debate é uma troca de pontos de vista, idéias e experiências embora expressas emocionalmente e sem lógica, mas sem privilegiar indivíduos, particulares ou posições?.

A análise das informações seguiu o processo de análise temática. A noção de tema está ligada a uma afirmação a respeito de determinado assunto. Ela comporta um feixe de relações e pode ser graficamente apresentada através de uma palavra, uma frase, um resumo ${ }^{10}$.

\section{ANÁLISE E DISCUSSÃO DOS RESULTADOS}

Da análise dos depoimentos dos nove sujeitos da pesquisa emergiu a categoria 0 Cuidado Especializado do Egresso da Residência do CEMO, abrangendo temas relacionados com: protocolo/tratamento/farmacologia/ansiedade, prescrição de enfermagem, orientação e experiência, esperança e complexidade A seguir contextualizaremos as falas que originaram esta categoria.

\section{TEMA 1 \\ Protocolo/ansiedade}

Ao tema um agregamos conceitos que se inter-relacionaram nas expressões dos sujeitos ao desenvolverem os cuidados ao paciente oncológico, naquilo que tem de mais peculiar.

[...] falamos [ao paciente] sobre a quimioterapia $e$ seus efeitos colaterais [...] (Rosa).

[...] muitas vezes pacientes e familiares perguntam sobre a quimioterapia, resultados de exames, transfusões, plaquetas, hemácias [...] (Violeta).

[...] conhecer o percurso das drogas quimioterápicas e seus efeitos colaterais [...] (Vitória Régia).
Conhecer os protocolos de cuidados, os tratamentos, a farmacologia e os resultados de exames para a implementação dos cuidados são de fundamental importância para 0 enfermeiro atuar com competência na sua especialidade. Em relação à farmacodinâmica e farmacocinética das drogas antineoplásicas, este é um conhecimento adquirido no ensino de pós-graduação lato sensu, neste caso, por ocasião do Programa de Residência em Enfermagem Oncológica do Instituto Nacional de Câncer, que propicia dentro de sua grade curricular conhecimentos específicos sobre as abordagens terapêuticas na área oncológica, e, especificamente, no campo de transplante de medula óssea, portanto, não se perdendo em soluções e diagnósticos infundados ${ }^{6}$.

[...] eu acho que o próprio paciente e a ansiedade dele e da família faz com que a gente fique mais próximo a ele [...] (Dália).

[...] o setor é fechado, o que contribui para um aumento da ansiedade e dependência da enfermagem [...] (Rosa).

No transplante de medula óssea, esta ansiedade é bastante evidente, em relação ao setor que é fechado, conforme fala da Rosa, ou seja, é considerada área restrita. Quando o paciente é submetido a alguma forma de restrição, no caso ambiental, 0 enfermeiro deve lançar mão de estratégias, dentre elas ajudar o paciente a aceitar o apoio de outras áreas, envolvendo, inclusive, as de ordem espiritual, a fim de melhorarem a qualidade do cuidado prestado aos pacientes ${ }^{6}$.

Estudos na área da enfermagem oncológica têm evidenciado que, em ambientes restritos e geradores de ansiedade, a atitude carinhosa da equipe é considerada um fator determinante de bem-estar. Isso porque ao ouvir com atenção, utilizar estratégias de descontração e irradiar alegria, estimulamos no paciente mudanças de atitude que the seja mais favorável ${ }^{13}$.

Os enfermeiros reconhecem nos pacientes e nos seus familiares algumas manifestações como, por exemplo, a ansiedade e a esperança. Ao vislumbrar estas necessidades que estão no campo da subjetividade, os enfermeiros ultrapassam os cuidados físicos e procuram num olhar mais profundo compreender o que se passa no âmago do paciente. Esta intersubjetividade que se expressa na relação cliente versus profissional possibilita ajuda mútua e o convívio salutar.

Situando esta perspectiva na história da oncologia, podese perceber que ela é circundada de aspectos que envolvem 0 emocional e o psicológico, por ser uma área onde o estigma toma vulto importante.

Ter domínio científico é indispensável para o trabalho dos enfermeiros, mas entender o paciente neste momento que sofre a dor e a desesperança que se fazem presentes em seu âmago é agregar valores importantes para uma assistência especializada em todos os seus aspectos e imprescindível para o relacionamento terapêutico.

\section{TEMA 2}

\section{Prescrição de Enfermagem}

A prescrição de enfermagem é primordial para o cuidado implementado no CEMO, sendo ressaltada pelo grupo como 
uma das ações a serem desenvolvidas pelos egressos, na medida em que a tomada de decisões é gerada pela competência profissional ${ }^{6-7}$.

Seguem os depoimentos abaixo:

[...] no banho de assento quem vai indicar é o enfermeiro! Os médicos mais antigos já sabem que isto é prescrição de enfermagem [...] (Rosa).

[...] se o paciente apresenta alguma alteração renal você já instituiu o balanço hídrico, a restrição hídrica em vez de 12, 06 ou a cada 04 horas [...] (Violeta).

[...] balanço hídrico quem institui é o enfermeiro, dependendo do protocolo[...] (Lírio).

Percebe-se que a prescrição de enfermagem como componente do processo de enfermagem é atividade privativa do enfermeiro. A prescrição de enfermagem é privativa do enfermeiro e deve ser implementada a fim de assegurar a continuidade do cuidado ao paciente ${ }^{11}$. Ratifica-se a sua importância pelo fato de ser um instrumento que possibilite a continuidade do cuidado e qualidade da assistência, especialmente levando-se em consideração, no caso em estudo, a complexidade do paciente oncológico e, em especial, do transplantado.

Verifica-se ainda pelos depoimentos que a prescrição de enfermagem, mesmo sendo atividade precípua do enfermeiro, também se apresenta como uma conquista dos enfermeiros, 0 que pode ser evidenciado no depoimento de Rosa quando diz: os médicos mais antigos já sabem que isto é prescrição de enfermagem. Esta constatação demonstra que os enfermeiros são reconhecidos pelos seus pares pela competência e pela incorporação de novos saberes e novas tecnologias, o que lhes dá visibilidade como profissionais especializados. Assim pontua como competência nuclear o cuidado baseado nos princípios da melhor prática, isto é, agir no sentido do melhor benefício para o paciente ${ }^{7}$.

Dentre os domínios dos cuidados de enfermagem estão a de diagnóstico, de acompanhamento e de monitorização do paciente ${ }^{6}$.

\section{TEMA 3}

\section{Orientação e Experiência}

Dentre as atividades desenvolvidas pelos egressos, a orientação se constitui em um dos principais pilares do tratamento de clientes do CEMO. A informação e o esclarecimento de dúvidas da clientela podem ser vislumbrados como um dos procedimentos que, sem de dúvida, muito auxiliarão no tratamento a ser realizado, como foi observado na fala a seguir:

[...] ele é encaminhando para o quarto onde fará o tratamento; apesar de já estar orientado, nós reforçamos, relembramos [...] (Rosa).

Ajudar o paciente a entender o processo em que está inserido é capacitá-lo a enfrentar o contexto de sua realidade. Expor ao paciente as etapas do tratamento, as possíveis complicações, muito contribuirá para uma melhor comunicação entre a equipe e para a adesão, por parte da clientela, ao tratamento e aos cuidados a serem implementados, facilitando, assim, a abordagem e a integração multidisciplinar.

Otimizar a participação do paciente para que este controle a sua própria cura e orientá-lo acerca das mudanças que acontecem, propondo novas escolhas, eliminando as antigas, guiando e educando, servindo de intermediário, caracterizase como um domínio que Benner denomina de função de ajuda ${ }^{6}$.

Assim, o que muitas vezes parece ser um cuidado simples pode ter uma especificidade que o torna complexo, implicando uma ação de enfermagem com alto grau de conhecimento ${ }^{6}$.

\section{TEMA 4}

\section{Esperança e Complexidade}

A esperança como um aspecto importante para se estabelecer o cuidado com os pacientes oncológicos foi apontada pelos depoentes como uma possibilidade de ajuda à clientela, como mostram os depoimentos a seguir:

[...] a primeira coisa que eu falo é de esperança; ele [o paciente] necessita mais de uma conversa do que um medicamento [...] (Dália).

[...] muitas vezes a resposta a ser dada não é aquela que ele quer ouvir, porque o prognóstico é desfavorável [...] (Vitória Régia).

Na rotina do trabalho do enfermeiro, os questionamentos dos clientes, diante do inusitado e do impacto que provoca, tornam-se carregados de desesperança e aflição, como exemplifica a fala de um paciente durante o tratamento quimioterápico: "Penso em desistir... se não fossem os vômitos e as náuseas, até que daria!".

Enfrentar algumas situações que caracterizem prognósticos desfavoráveis exigirá competências e habilidades para se comunicar ${ }^{6}$. A fala a seguir mostra a dimensão que se impõe: [...] muita vezes a resposta a ser dada não é aquela que ele quer ouvir, porque o prognóstico é desfavorável [...] (Vitória Régia).

Sabe-se que, na cultura ocidental, as pessoas são educadas para nascer e viver, e não para a finitude. Isto nos remete para o problema enfrentado na prática, quando o profissional da saúde entra em conflito sobre quando falar e como falar ao paciente.

A complexidade do paciente é outro tema que surge como característica para um cuidado especializado. É o que sugerem os depoimentos a seguir:

[...] cuidar de paciente transplantado abrange uma série de características pela complexidade deste paciente [...] (Orquídea).

[...] o nível de exigência de um paciente oncológico é diferenciado de outra clínica qualquer [...] (Azaléia).

A complexidade é fator imprescindível na prestação do cuidado ao paciente transplantado, é ponto de partida para o planejamento e a implementação de estratégias terapêuticas competentes. Portanto, um cuidado complexo deve se valer do conhecimento especializado, ou seja, de um conjunto de técnicas avançadas de avaliação para apresentar subsídios que contribuam com o cuidado multidisciplinar?

Sendo assim, todo o planejamento de enfermagem nas fases do transplante caracteriza uma atividade complexa necessitando de conhecimento especializado.

Há de se pontuar alguns fatores fundamentais que caracterizam a complexidade da assistência de enfermagem a pacientes em uma unidade de Transplante de Medula Óssea, 
como: as altas doses administradas de quimiorradiação que podem ser fatais, caso não haja um resgate com a nova medula infundida; as complicações sempre ocorrem simultaneamente e suas respectivas manifestações clínicas são semelhantes; uma complicação pode exacerbar ou causar outra complicação; a profilaxia, ou tratamento, para uma complicação pode ter que ser modificada ou até suspensa em função do desenvolvimento de outra complicação ${ }^{12}$.

Desse modo, o cuidado a ser prestado a este tipo de paciente requisita dos profissionais conhecimentos e habilidades compatíveis com o nível de atenção próprio dos especialistas.

\section{CONSIDERAÇÕES FINAIS}

0 cuidado de enfermagem prestado pelos egressos da Residência do INCA revela que os enfermeiros desenvolvem em sua atividades cotidianas ações no âmbito da objetividade e subjetividade. Isto ratifica os conceitos de especialista apresentados no referencial teórico.

\section{Referências}

1. Camargo TC. 0 ex-sistir feminino enfrentando a quimioterapia para o câncer de mama: um estudo de enfermagem na ótica de Martin Heidegger. [tese de doutorado]. Rio de Janeiro(RJ): Escola de Enfermagem Anna Nery/UFRJ; 2000.

2. Zancheta MS. Situações prioritárias na enfermagem em cancerologia. [dissertação de mestrado]. Rio de Janeiro (RJ): Escola de Enfermagem Anna Nery/UFRJ; 1990.

3. Instituto Nacional de Câncer- INCA. Regulamento do programa de residência em enfermagem oncológica. $1^{\text {a }}$ ed. Rio de Janeiro (RJ); 1986.

4. Lopes GT. A trajetória histórica da residência de enfermagem no Estado do Rio de Janeiro[tese de doutorado]. Rio de Janeiro (RJ): Escola de Enfermagem Anna Nery/UFRJ; 2001.

5. Instituto Nacional de Câncer- INCA. 0 Instituto: estrutura. [on-line] [citado 20 jun 2003c]. Disponível em: <www.inca.gov.br>

6. Benner P. De iniciado a perito. Coimbra ( PO) : Quarteto; 1984.

7. United Kingdom Council Center-UKCC. The council's standars for education and practice following registration. London(UK); 1994.

8. Merton RK. Teoria e estrutura social. México(ME): Fundo de Cultura Econômica; 1965.

9. Bauer MW, Gaskell G. Pesquisa qualitativa com texto, imagem e som: um manual prático. $2^{\text {a }}$ ed. Petrópolis ( RJ): Vozes; 2002.

10. Minayo MCS. 0 desafio do conhecimento: pesquisa qualitativa em saúde. 6ª ed. São Paulo (SP): Hucitec; 1999.
Atualmente no mundo do trabalho, a formação especializada tem sido bastante valorizada. Entretanto, já existe a cultura de que não basta somente conhecimento técnico para exercer atividades específicas, é necessário que outros valores sejam agregados para esta prática.

Assim, nesta perspectiva, os egressos desenvolvem atividades pertinentes e compatíveis com sua função especializada, como conhecer e utilizar procedimentos inerentes às drogas antineoplásicas e seus protocolos, realizar a prescrição de enfermagem visando ao atendimento da clientela em sua complexidade e especificidade, além de proporcionar apoio objetivando ajuda, não se limitando, portanto, às atividades somente tecnicistas, agregando valores humanísticos essenciais, já que o câncer é ainda uma doença que estigmatiza, trazendo embutida em si sentimentos de morte iminente, preconceitos e discriminação social. Tais características configuram que as enfermeiras desenvolvem o cuidado especializado, segundo 0 referencial teórico eleito para esta pesquisa.

11. Conselho Federal de Fnfermagem- COFEn. Resoluções. [on-line) [citado 15 maio 2003].Disponível em: <www.portalcofen.org.br>.

12. Forg R, Ballard B. Acute complications after bone marrow transplantation. Seminary in Oncology Nursing 1988; 4(1): 15-4.

13. Moura ACF, Moreira MC. A unidade de quimioterapia na perspectiva dos clientes: indicativos para gestão do ambiente na enfermagem oncológica. Esc Anna Nery Rev Enferm 2005 dez; 9 (3): 372-80.

\section{Sobre os Autores}

\section{Carlos Joelcio de Moraes Santana}

Mestre pela Faculdade Enfermagem da UERJ. Coordenador do Programa de Residência em Enfermagem Oncológica do Instituto Nacional de Câncer. Especialização em Enfermagem do Trabalho. Membro da Diretoria Executiva da Sociedade Brasileira de Enfermagem Oncológica (SBE0). Especialista em Enfermagem Oncológica pela Sociedade Brasileira de Enfermagem Oncológica (SBEO).

\section{Gertrudes Teixeira Lopes}

Professora Titular da Área de Pesquisa em Enfermagem da Faculdade de Enfermagem da UERJ. Doutora e Livre Docente em Enfermagem. PósDoutora na área de álcool e Drogas pela USP. Pesquisadora do CNPq. Membro da Diretoria Colegiada do Núcleo de Pesquisa em História da Enfermagem (MUPHEBRAS), da EEAN/UFRJ. Orientadora da dissertação. 